charities meet bi-monthly to discuss the progress of the needs assessment and consider how to work more closely together. Engagement activities for the needs assessment have included members of the public, patients and carers, schoolchildren, community groups, public and private health care providers, nursing home staff and residents with their families.

Conclusions Many of the issues encountered are similar to those in other settings and proximity does not always lead to closeness although personal connections are an advantage. There is some consistency so far in the views of participants that we aim for the right care, in the right place at the right time, although the small island setting presents some unique challenges.

\section{P-182 USING THE MODEL FOR IMPROVEMENT TO REDESIGN DAY THERAPY SERVICES}

Emily Stowe, Sarah Thompson, Carolanne Brannan. St Clare Hospice, Hastingwood, UK

\subsection{6/bmjspcare-2018-hospiceabs.207}

Background A review of Day Therapy (DT) services was required due to the challenges of increasing numbers of people with complex multi-morbidity needing services over a longer time period (Commission into the Future of Hospice Care, 2013). A need to provide more diverse and flexible services, able to meet the needs of a wider range of people was identified through service evaluation and user feedback. Transformational changes to services can be challenging to embed and maintain and the use of a framework can enable effective and sustainable service improvement (NHS England Sustainable Improvement and Horizon's Team, 2018).

Aims

- To evaluate the current model of day therapy

- To identify changes required to ensure services remain appropriate for our current and future patient population

- To use a service improvement framework to ensure changes are embedded effectively and sustained.

Methods The Model for Improvement (ACT Academy, 2018), was used by the DT staff team as a basis for redesigning and implementing the changes required. This model starts with three key questions, including 'what are we trying to accomplish?', followed by a Plan, Do, Study, Act (PDSA) cycle, allowing for continual analysis and improvement. The 'Fresh eyes' redesign tool was also used to facilitate viewing the redesign from the perspective of people using the service.

Results

- Evaluation of current service completed - identified elements that work well and those that are less effective

- Identified shared purpose for DT services: 'To provide a range of rehabilitative, holistic outpatient services to those with a variety of life- limiting conditions in a format that is individualised and flexible.'

- Identified proposed service changes, including outcome measurement and used PDSA cycle to implement

- Studied impact of changes using OACC data, attendance figures, user feedback.

Conclusion The Model for Improvement was an effective tool for planning and implementing change within the DT setting. It ensured focused planning and careful study which led to successful implementation of service improvement.

\section{P-183 USING IPOS TO EVALUATE THE IMPACT OF DAY THERAPY SERVICES}

Emily Stowe, Sarah Thompson, Carolanne Brannan. St Clare Hospice, Hastingwood, UK

\subsection{6/bmjspcare-2018-hospiceabs.208}

Background Outcome measures are designed to capture changes in health status as a consequence of clinical interventions (Witt, de Wolf-Linder, Dawkins et al., 2015). Measuring outcomes in palliative care can be challenging due to the progressive nature of the diseases people accessing services have. The OACC suite of outcome measures (Witt, de Wolf-Linder, Dawkins et al., 2015)has been in use in Day Therapy since 2015, in particular the Integrated Palliative Care Outcome Scale (IPOS) (https://pos-pal.org/).

Aims To audit the use of IPOS outcome measures in a day therapy setting. To assess the differences in reported IPOS scores between cancer and non-cancer patients. To evaluate any changes in IPOS scores between the beginning and end of Day Therapy sessions.

Methods A retrospective audit of the data all patients attending day therapy between March 2017 and January 2018 was completed and the following data was extracted from an electronic patient record database:

- Patient age

- Diagnosis

- Gender

- IPOS scores recorded for all Day Therapy attendances

- Dates of all Day Therapy attendances.

These data were then analysed using Excel spreadsheets. Results Between March 2017 and January 2018:

- 600 IPOS measures were completed by 112 different patients

- $53 \%$ of patients had a non-cancer diagnosis

- $28 \%$ of IPOS had one or more values missing

- The mean total IPOS score for cancer patients was 22.9

- The mean total IPOS score for non-cancer patients was 29.1

- Improvements were seen over time in all psychosocial, spiritual and practical domains of the IPOS

- Scores on the physical domains were maintained over time.

Conclusion A significant proportion of IPOS were not fully completed. Non-cancer patients scored on average higher than the cancer patients. Day Therapy appears to have a positive impact on psychosocial domains and maintains physical symptoms, which may be surprising given the progressive nature of the diseases those attending have. Methods to ensure completion of IPOS need to be identified.

\section{P-184 CREATIVE GROUP LEGACY PROJECT}

Kathryn Talbot-Vaux, Emily Stowe, Sarah Thompson. St Clare Hospice, Hastingwood, UK

\subsection{6/bmispcare-2018-hospiceabs.209}

Background Previous creative groups had been focused on creative exploration and distraction, however, it was decided that the focus of the creative groups should be more therapeutic, utilising the skills of the Occupational Therapists (OTs) and enabling patients to explore memories, thoughts and feelings. A new six-week legacy project was developed to enable patients to do this. 